\title{
Research on Interpolation Method of Random Hopped-Frequency in Synthetic Wideband Imaging
}

\author{
Junqun Liu, Dawei Lu and Jun Zhang \\ ATR Key Lab, National University of Defense Technology, Changsha, China
}

\begin{abstract}
Coherent pulse synthesis is commonly used in radar receiver for random hopped-frequency processing, which key is to make the frequency point of sampling to order and uniform arrangement, and then similar to the step frequency pulse signal for subsequent signal processing. In the view of numerical analysis in this paper, three different interpolation methods are used to analyze the frequency point of non-uniform arrangement. First, the corresponding mathematical model is given, and then the computation of these three methods are analyzed. Finally, simulation and comparison of high range resolution imaging for the echo signal of different signal and noise ratio with different interpolation methods.
\end{abstract}

Keywords-random hopped-frequency; non-uniform; interpolation; distance-imaging

\section{INTRODUCTION}

In Radar signal processing system, stepped frequency signal is popular with people because that it can obtain target's high range resolution $1 \mathrm{D} / 2 \mathrm{D}$ image by pulse echo coherent synthesis under the premise to reduce instantaneous bandwidth of the radar system. According to the waveform, stepped frequency is divided into order frequency step, COSTAS coding, step frequency modulation and pure random hopped frequency signal, which has advantage of good low probability of potential intercept anti interference and insensibility to Doppler, etc[1-3]. In recent years, it has been gradually applied in the random signal radar and the low intercept radar.

Random hopped frequency signal involved in this paper is pure random hopped frequency signal, and the radar signal carrier frequency between the pure completely jump at random within a certain range bandwidth. At this point, the echo pulse frequency will be completely change at random. The target echo can be regarded as random sampling frequency point of the target impulse response differenced from the COSTAS code[4]. This time even if the target echo reorders by the high and low order frequency point, the rearrangement of the sampling signal will still be the target response frequency domain of the nonuniform sampling. So if you want to use IFFT to synthetic distance for non-uniform sampling target response processing, it must also interpolate process with the non-uniform sampling according to the frequency point, transform the signal to the uniform frequency point within the bandwidth[5], and use IFFT imaging and subsequent coherent accumulation, etc.

This paper mainly investigates the different interpolation methods how to influence and analyze the imaging quality of HRRP under the circumstance of pure random hopped frequency. First, it gives the signal model of random hopped frequency radar imaging. Then gives the mathematical model of Lagrange interpolation, Hermite interpolation and spline interpolation in view of the non-uniform sampling signal caused by random. Finally, compares and analyses the imaging results of different interpolation methods by numerical simulation.

\section{IMAGING Model Of PURE RANDOM HopPeD FREQUENCY RADAR}

Using random hopped frequency pulse signal, the radar transmitter launch a string of $N$ narrow pulse, whose carrier frequency is random jumping at random within the range bandwidth. The launched of $\mathrm{m}(1 \leq m \leq N)$ pulse signal can be expressed as

$$
e_{m}(t)=A_{m} \cdot \operatorname{Rect}\left[\frac{t-(m-1) T}{\tau}\right] \cdot \exp \left(j 2 \pi f_{m} t+j \varphi_{0}\right)
$$

where $A_{m}$ is the amplitude of emission signal; $\operatorname{Rect}(\cdot)$ is expressed as the rectangle envelope; $f_{m}$ is the carrier frequency of $m$ pulse, $f_{0} \leq f_{n} \leq f_{0}+B$ and it is chosen by random; $\varphi_{0}$ is the initial pulse. In $N$ repetitive pulse cycle, the selection of $N$ frequency in the bandwidth will launch out in turn.

Supposed that there is a static target at the distance $R_{0}$, the vibration signal of coherent mix with the received signal before conversion of an intermediate frequency signal at the radar receiver. And according to the pulse width, sampled in the time of pulse receiver. Sampling sketch map in the target area shows in figure 1.

The echo signal $S_{m}(t)$ of $m^{\text {th }}$ pulse is expressed as

$$
\begin{aligned}
S_{m}(t) & =\sigma \cdot e_{m}\left(t-\frac{2 R_{0}}{c}\right) \\
& =B_{m} \cdot \operatorname{Rect}\left[\frac{t-2 R_{0} / c-(m-1) T}{\tau}\right] \cdot \exp \left[j 2 \pi f_{m}\left(t-\frac{2 R_{0}}{c}\right)+j \varphi_{0}\right]
\end{aligned}
$$

where $\sigma$ is the magnitude related to the scattering intensity of target; $B_{m}=\sigma A_{m}$ is amplitude of echo signal; $2 R_{0} / c$ is the delay time of target. After the coherent mixing echo signal, the random frequency point sampling sequence in the frequency domain can be obtained after sampling at the target echo. It is given by

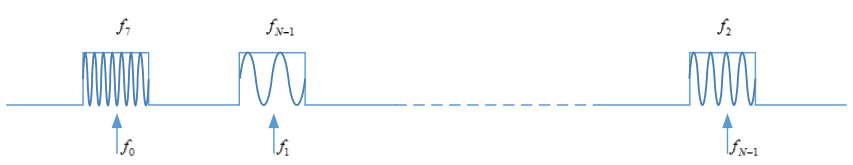

FIGURE I. SAMPLING SIGNAL IN THE RECEIVER 


$$
S(m)=S_{m}\left(t_{*}\right) \cdot\left(S_{m}^{\mathrm{REF}}\left(t_{*}\right)\right)^{*}=B_{m} \cdot \exp \left[-j \frac{4 \pi R_{0}}{c} f_{m}\right]
$$

where reference signal of $m$ pulse is $S_{m}{ }^{R E F}(t)=\exp \left(j 2 \pi f_{m} t+j \varphi_{0}\right)$. In order to get the target's high range image by using IDFT, it is necessary to rearrange the frequency point according to the ascending order, and get the non-uniform sampling sequence of frequency domain, which is expressed in a form as

$$
X\left(f_{\sigma_{m}}\right)=S\left(\sigma_{m}\right)=B_{\sigma_{m}} \cdot \exp \left[-j \frac{4 \pi R_{0}}{c} f_{\sigma_{m}}\right]
$$

where $f_{\sigma 1} \leq f_{\sigma 2} \leq \ldots \leq f_{\sigma N}, \sigma_{m}\{1,2, \ldots N\}$ is the frequency point after sort in term of ascending order. Since the sorting frequency interval is not necessarily equal, the IDFT process requires that the input sequences is uniform, so the interpolation method must be used to make the sorted sequence as the uniform frequency point sampling sequence.

\section{SAMPLING INTERPOLATION IN RADAR HOPPED FREQUENCY 'S FREQUENCY DOMAIN}

The sketch map and simulation of interpolation process of echo sequence is shown as figure 2. Common interpolation methods are Lagrange interpolation, Hermite interpolation and spline interpolation, etc.

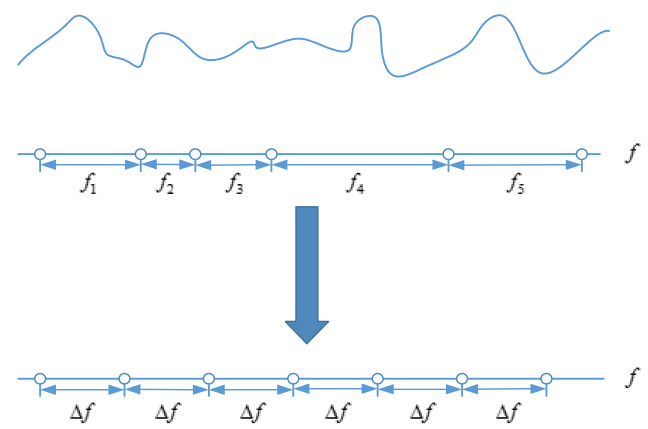

(a)

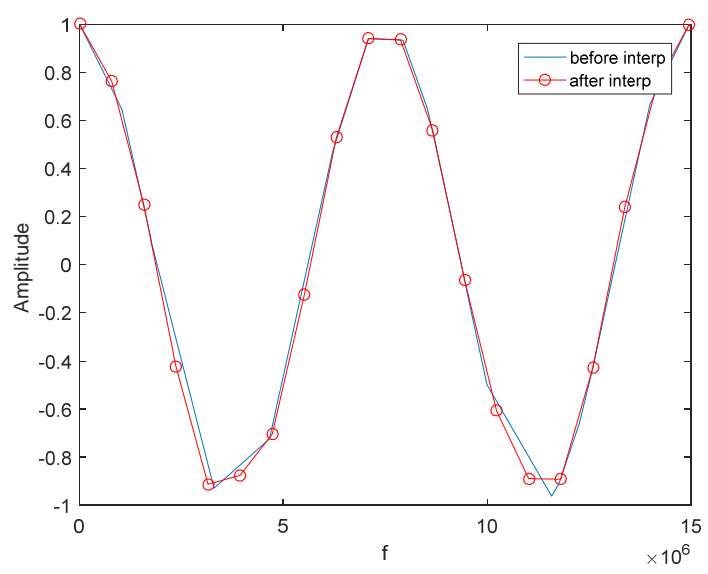

(b)

FIGURE II. THE PROCESS OF INTERPOLATION

\section{A. Lagrange Interpolation}

Linear and Newton interpolation are part of Lagrange interpolation. We assume that $f_{n}$ is a set of random frequency in the range of $\left[f_{\min }, f_{\max }\right], X_{n}$ is the sampling data of echo signal, which called $\left\{f_{n}, X_{n}\right\}_{n=1}^{N}$. The $N-1$ sub interpolation function can be constructed according to the $N$ sampling data, and shown as

$$
X(f)=\sum_{n=1}^{N} l_{n}(x) \cdot X\left(f_{\sigma_{m}}\right)=\sum_{n=1}^{N}\left(\prod_{\substack{j=1 \\ j \neq n}}^{N} \frac{f-f_{j}}{f_{n}-f_{j}}\right) \cdot X\left(f_{\sigma_{m}}\right)
$$

In fact, this is based on the known $f_{1}, f_{2}, \ldots, f_{N}$ to estimate the function $X(f)$, and let it close to the function $X\left(f_{\sigma_{m}}\right)$. The interpolation function $X(f)$ exists phenomenon of Runge when $N$ get its maximum. So it is used to take interpolation no more than three times, and then use the piecewise interpolation to estimate it.

According to the (5), we can get the three interpolation function of the echo signal in the first interval, as given by

$$
\begin{aligned}
X(f)=\frac{\left(f-f_{\sigma_{2}}\right)\left(f-f_{\sigma_{3}}\right)}{\left(f_{\sigma_{1}}-f_{\sigma_{2}}\right)\left(f_{\sigma_{1}}-f_{\sigma_{3}}\right)} X\left(f_{\sigma_{1}}\right)+\frac{\left(f-f_{\sigma_{1}}\right)\left(f-f_{\sigma_{3}}\right)}{\left(f_{\sigma_{2}}-f_{\sigma_{1}}\right)\left(f_{\sigma_{2}}-f_{\sigma_{3}}\right)} X\left(f_{\sigma_{2}}\right) \\
\quad+\frac{\left(f-f_{\sigma_{1}}\right)\left(f-f_{\sigma_{2}}\right)}{\left(f_{\sigma_{3}}-f_{\sigma_{1}}\right)\left(f_{\sigma_{3}}-f_{\sigma_{2}}\right)} X\left(f_{\sigma_{3}}\right)
\end{aligned}
$$

\section{B. Hermite Interpolation}

Sometimes it is hoped that the interpolation data can have a better smoothness at some extreme values. So it requires a higher interpolation function approximation, and this interpolation method is the use of the first derivative values at the interpolation points. By the (4), the first derivative of echo signal after sorted at the corresponding frequency is shown as

$$
X^{\prime}\left(f_{\sigma_{m}}\right)=-j \frac{4 \pi R}{c} \cdot B_{m} \exp \left(-j \frac{4 \pi R}{c} f_{\sigma_{m}}\right)
$$

The $N$ frequency point $f_{\sigma_{1}}, f_{\sigma_{2}}, \cdots, f_{\sigma_{n}}$ of echo signal function has corresponding function values as $X_{1}, X_{2}, \ldots, X_{n}$, and corresponding first derivation values as $X_{1}^{\prime}, X_{2}^{\prime}, \cdots, X_{n}^{\prime}$. So the Hermite interpolation polynomial of the function $X(f)$ of any $f$ in the interpolation interval is given by

$$
X(f)=\sum_{i=1}^{N} h_{i}\left[\left(f_{\sigma_{i}}-f\right)\left(2 \alpha_{i} X_{i}-X_{i}^{\prime}\right)+X_{i}\right]
$$

where

$$
h_{i}=\prod_{\substack{j=1 \\ j \neq i}}^{N}\left(\frac{f-f_{\sigma_{j}}}{f_{\sigma_{i}}-f_{\sigma_{j}}}\right)^{2} \quad \alpha_{i}=\sum_{\substack{j=1 \\ j \neq i}}^{N} \frac{1}{f_{\sigma_{i}}-f_{\sigma_{j}}}
$$

Similar to Lagrange interpolation, this method of interpolation nodes can't be too large, or the edge of the interpolation error will be greater. 


\section{Spline Interpolation}

When the radar signal transmitted, the choices of the number of pulses are less or more according to actual situation. The more pulse signals, the more corresponding sampling sequence points, interpolation function obtained by the interpolation method is polynomial of higher order degree, it will often appear fluctuation. In combination with the requirement of the whole smoothness of the interpolation function, the spline interpolation method is used. This method involves the function of the derivative of the problem, but also because of the function of the first order, the two order derivative continuous, the three order is not necessarily, so commonly used three interpolation.

Assume that echo signal after sorted has $n+1$ different frequency point $f_{\sigma_{m}}(m=0,1, \cdots, n)$ in the region $\left[f_{\min }, f_{\max }\right]$, and the corresponding amplitude value is $X_{m}$. When the function $X(f)$ satisfies that

1) In each sub region $\left[f_{j}, f_{j+1}\right] j \in[0,1, \cdots, n]$, it is a threedegree polynomial;

2) The first and second order derivatives are continuous in each of the internal node $f_{k}(k \in[1,2, \cdots, \mathrm{n}-1])$;

3) Have $X\left(f_{\sigma_{m}}\right)=X_{i}$ on each node.

Then $X(f)$ is called three spline interpolation function. This is to say that the three spline interpolation function, through all the interpolation nodes of the continuous and differentiable three degree polynomials constituted by $\mathrm{n}$ three-degree polynomials. Its expression is a piecewise function, and given by

$$
X(f)=\left\{\begin{array}{cc}
X_{1}(f) & f \in\left[f_{\sigma_{0}}, f_{\sigma_{1}}\right] \\
X_{2}(f) & f \in\left[f_{\sigma_{1}}, f_{\sigma_{2}}\right] \\
\vdots \\
X_{n}(f) & f \in\left[f_{\sigma_{n-1}}, f_{\sigma_{n}}\right]
\end{array}\right.
$$

According to the relevant information of the sampling sequence, the piecewise function $X_{m}(f)$ can be obtained by different methods, and the final interpolation function $X(f)$ can be obtained.

\section{Analysis of Operation}

1) In the Lagrange interpolation method, it is mainly the addition and multiplication. It need $4 N$ additions and $4 N^{2}$ multiplications for $N$ interpolation point.

2) In the Hermite interpolation method, which is similar to the Lagrange method, and need $4 N^{2}-4 N+3$ additions and $N(N-$ 1) $)^{2}$ multiplications.

3) In the spline interpolation method, the calculation of the number of piecewise function is different because of the selection of different methods. Analysis of its operation of the above methods are similar, it does not need to be analyzed in detail.

\section{Simulation Of High Resolution Imaging}

According to the methods previously described, based on the distance from the radar $R_{0}=20 \mathrm{~m}$ stationary target as an example. The paper simulates the high resolution range profiles. The simulation parameters are the signal bandwidth of $B=100 \mathrm{M}$, random sampling frequency point $N=128$, frequency points after interpolation are constant, echo sampling signal is plus-noise signal.

Through simulation experiments, the results obtained by different interpolation methods as show in figure 3 . We can see from figure 3, the data before and after the interpolation remained the same, but in some places the meander line, interpolation data under different interpolation methods have different deviation. In the figure4, the distance profile got under the three interpolation methods can be seen, and in the range of distance error, the correct range image of the target can be obtained under the three methods. However, the range imaging of the three different methods is not the same, such as the peak side lobe ratio (PSLR), the integral side lobe ratio (ISLR) and other indicators.

Let the signal-noise ratio(SNR) of echo signal change in the range of $[-20,100]$. The figure 5 is the result of the PSLR and ISLR change with SNR. By the theory, when the SNR is bigger, the signal quality is better, then PSLR and ISLR are also smaller.

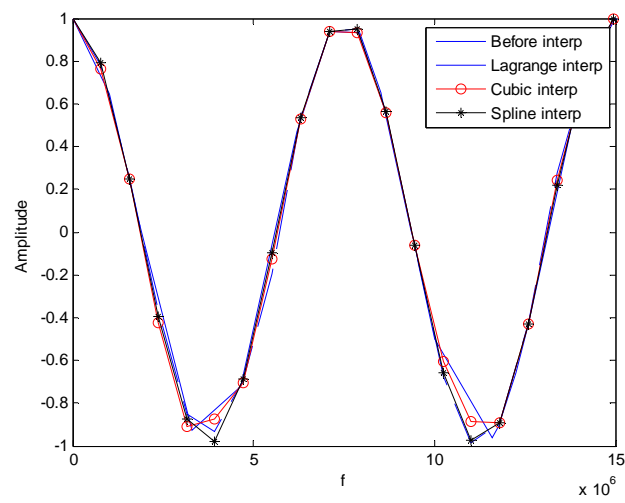

FIGURE III. COMPARISION DIAGRAM BEFOR AND AFTER INTERPOLATION

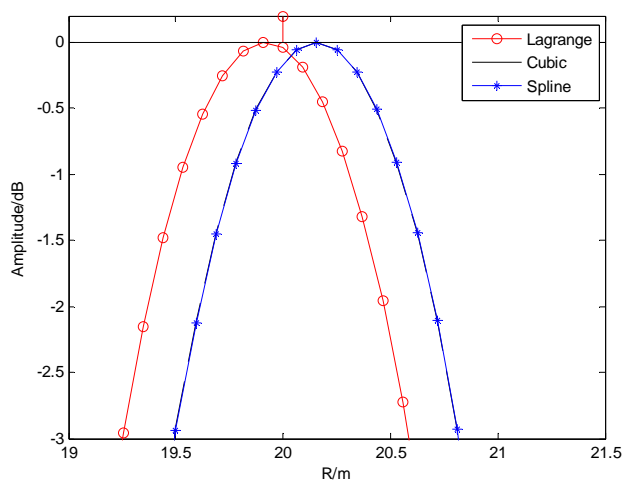

FIGURE IV. RANGE PROFILES AFTER DIFFERENT INTERPOLATION 


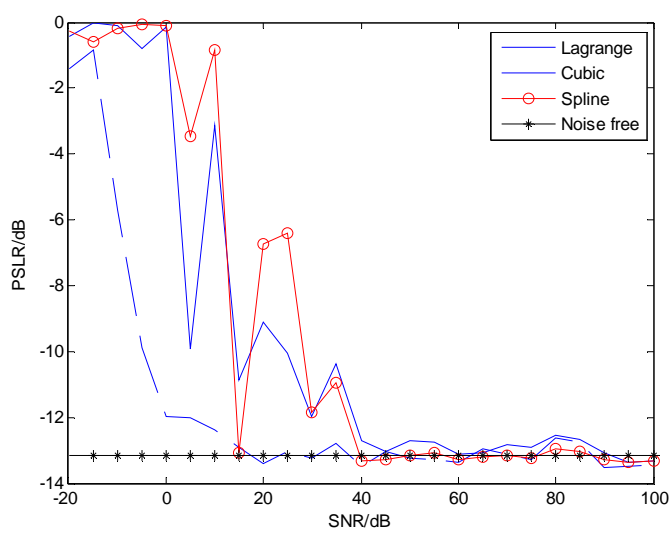

(a)

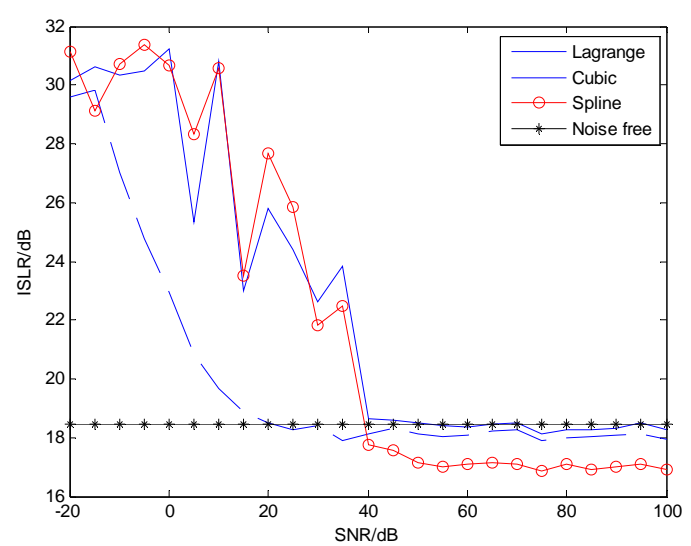

(b)

FIGURE V. PSLR AND ILSR CHANGE WITH SNR

In Figure 5, ISLR and PSLR are in line with the trend, and when the SNR reach a certain, PSLR tends to $-13 d B$, ISLR tends to $19 \mathrm{~dB}$, and they all accord with the need of correct imaging. Moreover, the anti-noise performance of the Hermite interpolation is better than the Lagrange interpolation and spline interpolation.

\section{CONCLUSION}

In this paper, the echo model of stationary target is presented, and the conventional method of synthesizing range profile is obtained according to the echo model. Focus on three kinds of interpolation methods commonly used in the research of synthetic imaging, Lagrange interpolation, Hermite interpolation, and spline interpolation. The basic principle and operation of the three interpolation methods are described respectively, and the imaging results are compared with the different signal-noise ratio. In practice, appropriate interpolation method should be selected according to the number of sampling frequency and the amount of computation.

\section{ACKNOWLEDGMENT}

This work was supported in part by the pre-research grant (71314092).

\section{REFERENCES}

[1] L. F. Ding, F. L. Geng, The Theory of Radar[M]. Xi'an:the press of xi'an university of electronic science and technology, 2002.

[2] X. H. Zheng, "Research of widthband radar signal of stepped sequence," The Technology of Fire Control Radar. China, vol. 1, pp. 16-19, 1998.

[3] Z. Liu, S. H. Zhang, "Analyse of high resolution radar signal with pulse synthetic," Guidance and Fuze. China, vol. 23, pp. 7-12, 2002.

[4] Y. L. Zhu, H. Q. Fan, and Z. Q. Lu, "One demensional high resolution doppler profile," Radar Science and Technology. China, vol. 8, pp. 32-37, 2010.

[5] Y. J. Zhang, H. Y. Su, and H. C. Zhu, "A novel waveform design for high range resolution radars," Telecommunication Engineering. China, vol. 48, pp. 48-53, 2008.

[6] W. D. Yu, S. M. Wu, "Comparision of servavel interpolation menthods for range-doppler profiles," Journal of Electronics and Information Technology. China, vol. 23, pp. 308-312, 2001.

[7] Z. C. Qi, Numeric analyse and application[M], Changsha: the press of national university of defense technology, 1995. 